\title{
The Magnitude of MTB and Rifampicin Resistance MTB Using Xpert-MTB/RIF Assay Among Tuberculosis Suspected Patients in Gedeo Zone, Southern Ethiopia
}

\author{
Kuma Diriba (D) \\ Ephrem Awulachew' \\ Gemechu Churiso² \\ 'Department of Medical Laboratory \\ Sciences, Health Science and Medical \\ College, Dilla University, Dilla, Ethiopia; \\ ${ }^{2}$ Department of Medical Laboratory \\ Sciences, Immunology Unit, Health \\ Science and Medical College, Dilla \\ University, Dilla, Ethiopia
}

Background: Tuberculosis (TB) remains a major global health problem causing death among millions of people each year. The new barrier that challenges the control of tuberculosis is the emerging and the increasing number of drug-resistant TB that becomes a world concern. This study aimed to determine the magnitude of rifampicin-resistant Mycobacterium tuberculosis (RR-MTB) among presumptive TB patients attending Dilla University Referral Hospital, Gedeo Zone, Ethiopia.

Methods: A retrospective cross-sectional study was conducted at Dilla University Referral Hospital from January 2014 to December 2020. Sputum results were done using Xpert MTB/ RIF assay and other necessary data were collected from the registration logbooks using a standardized data extraction format and analyzed using SPSS version 23 statistical software. Results: A total of 17,745 presumptive TB patients were included, of which $62.2 \%$ were males. The overall prevalence of Mycobacterium tuberculosis (MTB) was $11.8 \%$, of which $5.1 \%$ were confirmed to have RR-MTB. Extra-pulmonary TB was reported in $1.5 \%$ of the study participants. The highest prevalence of MTB and RR-MTB was recorded in 2017 with a prevalence of $20.1 \%$ and $8.5 \%$, respectively. All age groups were significantly associated with a higher prevalence of MTB $(p<0.036)$. TB patients with a history of previous treatment and HIV positive were significantly associated with MTB $(P<0.021)$, while RR-MTB was only significantly associated with patients with a history of previous treatment $(P<0.018)$.

Conclusion: A high magnitude of MTB and RR-MTB was reported among TB patients with HIV and a history of previous treatment. Therefore, coordinated efforts should be applied to the improvement of treatment adherence of known TB cases, and appropriate control and prevention methods to reduce the emergence and increase of MTB and RR-MTB cases.

Keywords: presumptive TB, MTB, RR-MTB, Xpert MTB/RIF assay, previous treatment, HIV/TB

\section{Introduction and Background}

Tuberculosis is an airborne disease resulting from Mycobacterium tuberculosis (M. tuberculosis). It typically affects the lungs and can also spread to other organs causing extra-pulmonary TB. ${ }^{1}$ The World Health Organization (WHO) recently announced that TB remains a major global health problem causing deaths among millions of people each year. TB is the ninth leading cause of death worldwide and the leading cause from a single infectious agent, ranking above HIV. Globally, TB
Correspondence: Kuma Diriba Tel +251913384550

Email kumadiriba47@gmail.com 
kills almost three people every minute. ${ }^{2}$ In 2019 , WHO estimated 9.6 million people developed TB and 1.2 million died of the disease. ${ }^{2,3}$ TB mortality and incidence rate are falling at about $3 \%$ and $2 \%$ per year worldwide, respectively. ${ }^{4}$

The main new barrier that challenges the control of TB is the high burden of multidrug-resistant TB (MDR-TB). In 2017, WHO estimated 580,000 new cases of RR-MTB worldwide, making them eligible for MDR-TB treatment. ${ }^{5}$ Of these, around 475,600 had MDR-TB. In total, 3.5\% of new TB cases and $18 \%$ of patients with a history of previous treatment were found to be MDR-TB. ${ }^{6}$ This makes the MDR strain become a prime global concern. WHO has been targeting an end TB strategy based on an assessment of the TB epidemic and progress in TB diagnosis, treatment, and prevention efforts. This shift in the approach to TB control, which includes among its 2030 targets $(90 \%$ TB case detection and treatment) including in high-risk populations, and a cure rate of $90 \%$ of detected TB cases. ${ }^{7}$

The developments of bacterial resistance to specific antibiotics are becoming an important consideration for clinicians. The emerging and increasing drug-resistant TB has resulted in a great challenge in the Africa region, subSaharan Africa (SSA) in particular. SSA stands the burden of both very high TB incidence and prevalence rates in the world and represents $14 \%$ of the global burden of new MDR-TB cases. ${ }^{8}$ Ethiopia ranks 10th among the high TBpandemic countries and 15th among the 27 high-MDR-TB countries in the world and 3rd in Africa following South Africa and Nigeria, ${ }^{9}$ with more than 5000 estimated MDRTB patients each year. ${ }^{10,11}$ The prevalence of MDR-TB in Ethiopia is $7.24 \%{ }^{12}$ Different studies in Ethiopia reported that patients with a history of previous treatments were significantly associated with MDR-MTB infection. ${ }^{13}$

The early diagnosis and treatment of TB patients are mandatory to reduce transmission of the disease. Millions of people are diagnosed and successfully treated for TB each year, averting millions of deaths, but there are still large gaps in detection and treatment. As to our knowledge, there are no data regarding the prevalence of MTB, RRMTB, and associated risk factors in this particular study area. Therefore, the current study provides updated information on the prevalence of MTB, RR-MTB, and associated risk factors to decide health priorities, to allocate resources, and to monitor the emergence of resistance for planning effective use of anti-TB drugs. It also serves as preliminary information for health programmers to give special attention and design a package in the national TB control program that addresses such areas where thousands of people live in an overcrowded area. Hence, this study aimed to assess the magnitude of MTB and RR-MTB among presumptive TB patients in Gedeo Zone, Southern Ethiopia.

\section{Methods}

\section{Study Design and Study Population Study Setting}

The study was conducted in the Gedeo Zone, which is located in the southern direction of Ethiopia with a total estimated population of $1,694,868$ according to the 2007 population census conducted by the Central Statistical Agency of Ethiopia (data are from the Zonal health office). Gedeo Zone is found at a distance of $85 \mathrm{~km}$ from Hawassa and $365 \mathrm{~km}$ from Addis Ababa, the capital city of Ethiopia. It is located in the kola agro-ecological zone with an altitude of $1400 \mathrm{~km}$ above sea level and annual temperature ranging from $22{ }^{\circ} \mathrm{C}$ to $29^{\circ} \mathrm{C}$. ${ }^{14}$

A retrospective cross-sectional study design was used to collect seven-year data from January 2014 to December 2020 at Dilla University Referral Hospital. The study populations were all pulmonary TB suspected patients who visited the selected health institution between January 2014 and December 2020.

\section{Eligibility Criteria}

Inclusion criteria: we included all presumptive pulmonary TB patients with full information like age, sex, Xpert MTB/ RIF assay results, HIV status, and previous TB treatment history results recorded on the registration logbook.

Exclusion criteria: study participant data with incomplete information like age, gender, Xpert MTB/RIF invalid, indeterminate Xpert MTB/RIF results, HIV status, and TB treatment history results recorded on the logbook.

\section{Variables}

Outcome variable: MTB and RR-MTB.

Independent variables: all included determinants (age, gender, HIV status, and TB treatment history).

\section{Operational Definitions}

New cases: patients who have never been treated for TB before.

Relapse cases: patients who were declared cured or treatment completed by a physician but who report back to the health service and are now found to be sputum smear or culture positive. 
Lost to follow up: a TB patient who did not start treatment or whose treatment was interrupted for 2 consecutive months or more.

Failure case: a TB patient whose sputum smear or culture is positive at month 5 or later treatment.

MDR-TB: TB that does not respond to at least isoniazid and rifampicin, the most important first-line anti-TB drugs.

Rifampicin-resistant TB (RR-TB): resistance to rifampicin detected using genotypic or phenotypic methods with or without resistance to other first-line anti-TB drugs.

Default: patients who were not taking anti-TB drugs for 2 months or more, consecutively after starting treatment.

\section{Data Collection and Laboratory Processing}

Sociodemographic characteristics of study participants (age and sex) and clinical feature-related data (Gene Xpert MTB/RIF assay results, HIV antibody test results, and previous TB patient treatment history results) were collected using a pretested structured data extraction sheet by a trained laboratory technologist from the TB registration book at the TB laboratory and TB clinic.

\section{Laboratory Processing}

Those patients identified with signs and symptoms of pulmonary tuberculosis were ordered to bring a single sputum sample for the diagnosis of TB using the Xpert MTB/RIF assay (Cepheid, CA, USA). Briefly, $2 \mathrm{~mL}$ of Gene Xpert MTB/RIF sample reagent buffer was added to $1 \mathrm{~mL}$ of sputum specimen using a sterile pipette. The closed specimen container was manually agitated twice for 15 seconds and allowed to stand at room temperature for $10 \mathrm{~min}$ and again vortexes after $10 \mathrm{~min}$ and allowed to stand for $5 \mathrm{~min}$ and then $2 \mathrm{~mL}$ of the inactivated material was transferred to the test cartridge and the cartridge was then loaded into Gene Xpert device. Finally, the results were interpreted by the Gene Xpert diagnosis system from the measured fluorescent signals and displayed automatically after 2 hours. Rapid HIV test was done according to the national HIV test algorithm.

\section{Data Processing and Analysis}

After all demographic data and patients' history were collected from the registration book, data entry, data analysis, and data cleaning were done using Epi-Data 3.1 and SPSS version 23.0 software. Frequency count and percentage were used to present the findings. Prevalence figures were calculated for the total study population and separately by clinical features of the disease. Association factors were assessed by bivariate logistic regression and multivariate logistic regression. Multivariate analysis was run by selecting those variables that appeared to have a $P$-value of $<0.25$ in the bivariate analysis to control the confounding effect of different variables. $P$-value of $<0.05$ was considered as statistically significant.

\section{Ethical Consideration}

The protocol for patient recruitment and participation in the study followed the principles of the Declaration of Helsinki and was approved by the Dilla University Health Research Ethics Review Committee under the protocol unique number 005/21-01. In the current study, sociodemographic and clinical data of TB suspected patients were collected retrospectively from medical records after getting permission to conduct the study from Dilla University Referral Hospital medical director and respective departments. Informed consent was not applicable for study participants since the study was retrospective, and we have used the clinical data obtained from routine medical services. Strict confidentiality was maintained by removing all patient identifiers and only code numbers were used throughout the study.

\section{Results}

\section{Sociodemographic and Clinical Features of Study Participants}

A total of 17,745 study participants suspected with TB and who have given sputum samples which had complete data were included in this study, out of which $62.2 \%$ were male and $37.8 \%$ were female with a sex distribution ratio of 1.6:1. The age of the study participants ranged from 1 year to 95 years with a median age of 37 (interquartile range: 24.7-49.3 years). The majority of study subjects were between the age group of 30-44 years which accounts for $30.6 \%$, followed by the 15-29 year age group accounting for $24.4 \%$. The majority of the study participants were new cases by accounting for $87 \%$ of the total participants. Among those participants tested for HIV, 52.4\% were negative while only $2.5 \%$ were HIV positive. Out of TB suspected cases, MTB was isolated from 2090 giving an overall prevalence of $11.8 \%$ while the prevalence of EPTB was $1.5 \%$. The prevalence of RR-MTB was $5.1 \%$ (Table 1). 
Table I Sociodemographic, Clinical Characteristics and MTB results Among TB Suspected Patients in Southern Ethiopia, 2014-2020 ( $N=17,745)$

\begin{tabular}{|c|c|c|c|}
\hline Variables & Categories & Frequency & Percentage (\%) \\
\hline \multirow[t]{2}{*}{ Gender } & Male & 11,029 & 62.2 \\
\hline & Female & 6716 & 37.8 \\
\hline \multirow{6}{*}{$\begin{array}{l}\text { Age } \\
\text { categories }\end{array}$} & $<15$ & 1154 & 6.5 \\
\hline & $15-29$ & 4323 & 24.4 \\
\hline & $30-44$ & 5429 & 30.6 \\
\hline & $45-59$ & 3634 & 20.5 \\
\hline & $60-74$ & 2758 & 15.5 \\
\hline & $>75$ & 447 & 2.5 \\
\hline \multirow[t]{2}{*}{ MTB result } & Detected & 2090 & 11.8 \\
\hline & Not detected & 15,655 & 88.2 \\
\hline \multirow[t]{2}{*}{ Types of TB } & PTB & 2090 & 11.8 \\
\hline & EPTB & 268 & 1.5 \\
\hline \multirow{7}{*}{$\begin{array}{l}\text { History of TB } \\
\text { treatment }\end{array}$} & New case & 15,434 & 87.0 \\
\hline & Relapse & 674 & 3.8 \\
\hline & Lost to & 1306 & 7.4 \\
\hline & follow up & & \\
\hline & Failure & 112 & 0.6 \\
\hline & Transfer & 156 & 0.9 \\
\hline & Default & 63 & 0.4 \\
\hline \multirow{3}{*}{$\begin{array}{l}\text { HIV status for } \\
\text { antibody test }\end{array}$} & Positive & 436 & 2.5 \\
\hline & Negative & 9307 & 52.4 \\
\hline & Unknown & 8002 & 45.1 \\
\hline \multirow{3}{*}{$\begin{array}{l}\text { RR-MTB } \\
\text { result }\end{array}$} & RR-MTB & 107 & 5.1 \\
\hline & detected & & \\
\hline & $\begin{array}{l}\text { RR-MTB not } \\
\text { detected }\end{array}$ & 1983 & 94.9 \\
\hline
\end{tabular}

\section{Prevalence of MTB and RR-MTB by Years}

We tried to compare the prevalence of MTB and RR-MTB by year (as indicated in Figure 1) in which the study participants were treated. Our study showed that the highest prevalence of MTB was recorded in 2017, followed by 2020 and 2018 with the prevalence of $20.1 \%, 16.2 \%$, and $10.4 \%$, respectively. The least prevalence of MTB was reported in 2019 with a prevalence of $6.7 \%$. Similarly, the highest prevalence of RR-MTB was reported in 2017 with a prevalence of $8.5 \%$ followed by 2019 and 2018 with the prevalence of $8.0 \%$ and $7.1 \%$, while the least prevalence was reported in 2016 and 2020 with a prevalence of $2.2 \%$ and $2.9 \%$ (Figure 1).

\section{Associated Risk Factors of MTB Infections}

In the current study, a higher prevalence of MTB was reported in males $(58.6 \%)$, but there is no significant association with MTB ( $p$-value $>0.05$ ). The occurrence of MTB was significantly associated with different age groups ( $p$-value < 0.035). The likelihood of having MTB showed a decreasing trend as the age of study participants increases. Regarding treatment history, relapsed cases and treatment failure were significantly associated with the occurrence of MTB ( $p$-value < 0.001). The likelihood of having MTB showed 1.71 times higher in HIV positive than in HIV negative patients (AOR: 1.71; 95\% CI: 1.39-2.81, $p<$ 0.001) (Table 2).

\section{Associated Risk Factors of RR-MTB}

Among the total MTB confirmed patients, 107 (5.11\%) of them were positive for RR-MTB. In this study, there is no association between sex and different age groups. Regarding the treatment history, the likelihood of patients with relapsed cases $(\mathrm{AOR}=2.12 ; 95 \%$ CI $1.55-2.43, p=$ 0.01 ), failed cases $(\mathrm{AOR}=1.42 ; 95 \%$ CI $1.22-2.79, p=$ $0.031)$, and default cases (AOR $=1.13 ; 95 \%$ CI 1.11, 1.64, $p$-value $=0.023)$ were more likely to have RR-MTB compared to the new cases (Table 3).

\section{Discussion}

Data on the local epidemiology of MTB and RR-MTB and associated risk factors were useful for the prevention and control of MTB, but limited data were available in the study area. In our study, the overall prevalence of MTB and RRMTB was $11.8 \%$ and $5.1 \%$, respectively. In the current study, the prevalence of MTB was in line with previous reports from Amhara, $11 \%,{ }^{15}$ Addis Ababa, $12.5 \%,{ }^{16}$ South Africa, $13 \%,{ }^{17}$ and Korea, $13.8 \% .{ }^{18}$ However, our finding was lower than studies conducted in different parts of Ethiopia with prevalence ranging from 15.11 to $60.4 \%,{ }^{15,19-24}$ Nigeria, $22.9 \%,{ }^{25}$ Congo, $79.1 \%,{ }^{26}$ and Togo, $57 \%{ }^{27}$ In contrast to our study, however, the studies conducted in Uganda, $5.5 \%,{ }^{28}$ Addis Ababa, $6 \%,{ }^{29}$ and Oromia region, $3.8 \%,{ }^{30}$ were lower than our current study. The difference might be due to variation in methodological techniques (culture vs Xpert), study participants, study period, sample size, geography, TB control, and prevention practices. In our study, there is no significant association between males and females. But different previous studies reported that being male was a risk factor for MTB and MDR-TB development. ${ }^{31-33}$

In this study, participants of age groups between 15 and 29 years were more infected by MTB compared to other age groups $(\mathrm{AOR}=2.31 ; 95 \%$ CI $2.08-2.51, p<0.001)$. Similarly, different studies reported a higher prevalence of MTB in age groups ranging from 16 to 34 years, ${ }^{20,23}$ while 
MTB-RR/MTB Vs Year Sample Processed

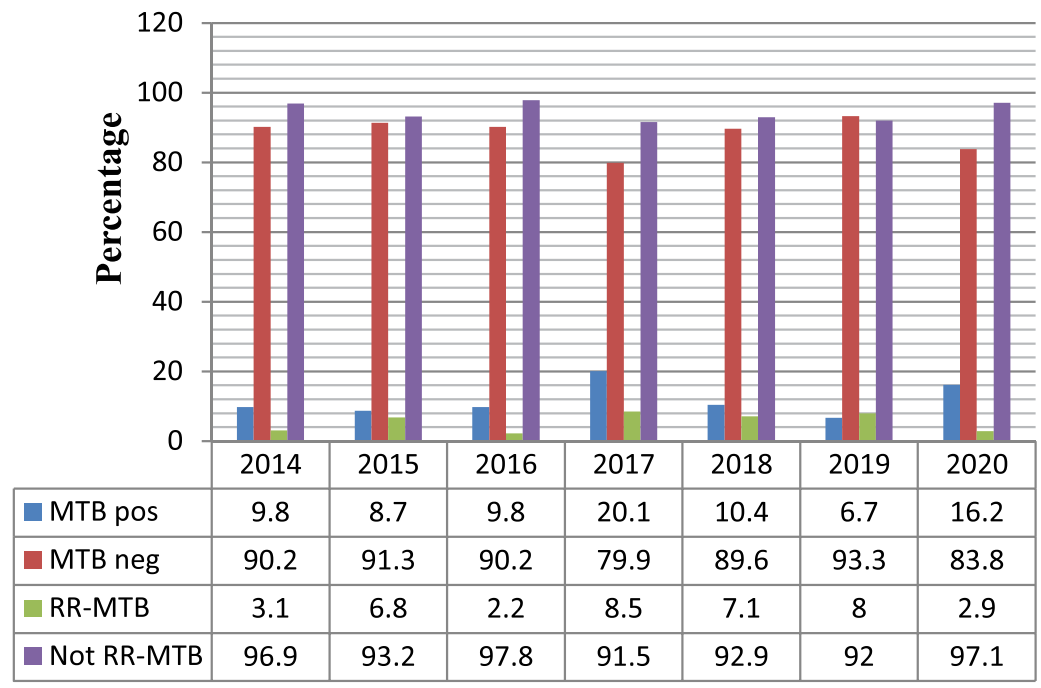

MTB pos

MTB neg

RR-MTB

Not RR-MTB

Figure I The magnitude of MTB and RR-MTB by years in Southern Ethiopia, 20I4-2020 (N = 17,745)

other studies conducted in different areas reported no association between age and TB infection. ${ }^{34-37}$ Our study showed no significant association between gender and TB patients. However, studies conducted in the Philippines ${ }^{38}$ and North Sudan, ${ }^{39}$ and elsewhere, ${ }^{19}$ reported that females were less likely to have MTB compared to males. The explanation for enhanced MTB in males could be probably due to males are mainly involved in outdoor activities and

Table 2 The Magnitude of MTB in Different Study Participant Categories (Gender, Age, Treatment History, and HIV Status) in Southern Ethiopia, 2014-2020 ( $N=17,745)$

\begin{tabular}{|c|c|c|c|c|c|c|}
\hline & MTB Pos N (\%) & MTB Neg N (\%) & COR $(95 \% \mathrm{Cl})$ & $P$-value & AOR $(95 \% \mathrm{Cl})$ & $P$-value \\
\hline \multicolumn{7}{|l|}{ Gender } \\
\hline Male & I224 (58.6) & $9805(62.6)$ & Ref & & Ref & \\
\hline Female & $866(41.4)$ & $5850(37.4)$ & $0.95(0.76-1.05)$ & 0.241 & $0.95(0.76-1.05)$ & 0.241 \\
\hline \multicolumn{7}{|c|}{ Age categories } \\
\hline$<15$ & $132(6.3)$ & $1022(6.5)$ & Ref & & Ref & \\
\hline $15-29$ & $605(28.9)$ & $3718(23.7)$ & $2.45(2.13-2.75)$ & $<0.00 I^{*}$ & $2.3 \mathrm{I}(2.08-2.5 \mathrm{I})$ & $<0.001 *$ \\
\hline $30-44$ & $554(26.5)$ & $4875(31.1)$ & $0.25(0.13-0.55)$ & $0.021 *$ & $0.24(0.12-0.54)$ & $0.021 *$ \\
\hline $45-59$ & $472(22.6)$ & $3162(20.2)$ & $1.25(1.14-1.55)$ & $0.011 *$ & $1.23(1.06-1.39)$ & $0.011^{*}$ \\
\hline $60-74$ & $281(13.4)$ & 2477 (15.8) & $1.21(1.11-1.43)$ & $0.033^{*}$ & $1.21(1.11-1.43)$ & $0.032 *$ \\
\hline$>75$ & $46(2.2)$ & $40 I(2.6)$ & $0.24(0.12-0.46)$ & $0.036^{*}$ & $0.23(0.11-0.44)$ & $0.035^{*}$ \\
\hline \multicolumn{7}{|c|}{ History of TB treatment } \\
\hline New case & $1940(92.8)$ & 13,494 (86.2) & Ref & & Ref & \\
\hline Relapse & $77(3.7)$ & $597(3.8)$ & $2.03(2.0 \mathrm{I}-2.45)$ & $<0.00 I^{*}$ & $2.02(2.0 \mathrm{I}-2.43)$ & $<0.001 *$ \\
\hline Lost & $23(1.1)$ & I 283 (8.2) & $1.24(0.93-2.77)$ & 0.273 & $1.22(0.68-2.34)$ & 0.272 \\
\hline Failure & $22(I . I)$ & $90(0.6)$ & $2.47(1.23-2.95)$ & $<0.00 I^{*}$ & $2.42(1.22-2.79)$ & $<0.001 *$ \\
\hline Transfer & $22(1.1)$ & $134(0.9)$ & $0.67(0.31-1.98)$ & 0.263 & $0.65(0.30-1.91)$ & 0.261 \\
\hline Default & $6(0.3)$ & $57(0.4)$ & $1.14(1.12-2.66)$ & $0.021^{*}$ & $1.13(1.11-2.64)$ & $0.02 I^{*}$ \\
\hline \multicolumn{7}{|c|}{ HIV status } \\
\hline Positive & $160(36.7)$ & $276(63.3)$ & $1.73(1.43-2.85)$ & $<0.00 I^{*}$ & I.7I (I.39-2.8I) & $<0.00 I^{*}$ \\
\hline Negative & $1498(16.1)$ & 7809 (83.9) & $0.57(0.26-1.88)$ & 0.224 & $0.55(0.23-1.79)$ & 0.222 \\
\hline Unknown & $432(5.4)$ & 7570 (94.6) & Ref & & Ref & \\
\hline
\end{tabular}

Note: *Statistically significant. 
Table 3 The Magnitude of RR-MTB in Different Study Participant Categories (Gender, Age, Treatment History, and HIV Status) in Southern Ethiopia, 20I4-2020 ( $N=2090)$

\begin{tabular}{|c|c|c|c|c|c|c|}
\hline Variable & RR-MTB Detected N (\%) & RR-MTB Not N (\%) & COR $(95 \% \mathrm{CI})$ & $P$-value & AOR $(95 \% \mathrm{Cl})$ & $P$-value \\
\hline \multicolumn{7}{|l|}{ Gender } \\
\hline Male & $74(69.2)$ & $1150(58.0)$ & Ref & & Ref & \\
\hline Female & $33(30.8)$ & $833(42.0)$ & I.3। (0.76-I.35) & 0.241 & $1.31(0.76-1.45)$ & 0.242 \\
\hline \multicolumn{7}{|c|}{ Age categories } \\
\hline$<15$ & $4(3.7$ & $128(6.5)$ & I.II (0.63-I.76) & 0.66 & I.II (0.64-I.76) & 0.67 \\
\hline $15-29$ & $27(25.2)$ & $581(29.3)$ & $1.22(0.34-3.75)$ & 0.43 & $1.23(0.34-3.76)$ & 0.42 \\
\hline $30-44$ & $31(29.0)$ & $523(26.4)$ & $1.15(0.14-1.53)$ & 0.23 & $1.17(0.15-1.63)$ & 0.24 \\
\hline $45-59$ & $25(23.4)$ & $443(22.3)$ & $0.42(0.21-1.33)$ & 0.36 & $0.42(0.21-1.33)$ & 0.36 \\
\hline $60-74$ & $18(16.8)$ & $263(13.3)$ & $1.24(0.52-1.46)$ & 0.31 & $1.25(0.52-1.46)$ & 0.31 \\
\hline$>75$ & $2(1.9)$ & $45(2.3)$ & I.24 (0.32-2.46) & 0.42 & $1.25(0.32-2.45)$ & 0.42 \\
\hline \multicolumn{7}{|c|}{ History of TB treatment } \\
\hline New case & $62(57.9)$ & 1878 (94.7) & Ref & & Ref & \\
\hline Relapse & 17 (I5.9) & $60(3.3)$ & $2.17(1.58-2.45)$ & $0.011 *$ & $2.16(1.55-2.43)$ & $0.010^{*}$ \\
\hline Lost & $5(4.7)$ & $18(0.9)$ & $1.24(0.93-3.77)$ & 0.052 & $1.22(0.68-3.34)$ & 0.051 \\
\hline Failure & $13(12.2)$ & $9(0.5)$ & $3.47(2.23-4.95)$ & $<0.001 *$ & $3.42(2.22-4.79)$ & $<0.00 I^{*}$ \\
\hline Transfer & $6(5.6)$ & $16(0.8)$ & $0.67(0.31-1.98)$ & 0.263 & $0.65(0.30-1.91)$ & 0.261 \\
\hline Default & $4(3.7)$ & $2(0.1)$ & $2.14(1.12-3.66)$ & $0.019 *$ & $2.13(1.11-3.64)$ & $0.018^{*}$ \\
\hline \multicolumn{7}{|c|}{ HIV antibody test } \\
\hline Positive & $61(57.0)$ & $99(5.0)$ & I.73 (0.39-2.85) & 0.430 & I.7I (0.39-2.8I) & 0.421 \\
\hline Negative & $44(4 \mid . I)$ & I454 (73.3) & $0.57(0.26-1.88)$ & 0.524 & $0.55(0.23-1.79)$ & 0.522 \\
\hline Unknown & $2(1.9)$ & $430(21.7)$ & Ref & & Ref & \\
\hline
\end{tabular}

Note: *Statistically significant.

have more frequent contact with TB patients while females usually stay at home.

In our study, participants who had a history of previous treatment (failure, relapse, and default) were more infected by MTB compared to new cases. This result is in agreement with previous studies conducted in Ethiopia. ${ }^{15,23,40}$ Enhanced frequency of MTB in patients having a history of previous treatment indicates high transmission of TB infection in the community. So, serious emphasis should be given for the prevention and control to limit its transmission.

In the current study, the prevalence of RR-MTB among patients suffering from TB infection was $5.1 \%$. This result is nearly comparable with other previous studies conducted in Nigeria, 7.3\%, ${ }^{41}$ Uganda, 5.7\%, ${ }^{42}$ and Ethiopia, $8.3 \%{ }^{43}$ In contrast to our finding, however, previous studies conducted in different parts of Ethiopia, 11-54\%, ${ }^{11,22,24,29,44}$ Nigeria, $14.7 \%,{ }^{45}$ Congo, $42.2 \%,{ }^{26}$ and in Togo, $24 \%,{ }^{27}$ reported a higher prevalence of RR-MTB than our study. On the other hand, a lower prevalence was reported by studies conducted in other parts of Ethiopia, 1.2-4.7\%, ${ }^{20,23,30,34,46}$ and the WHO report of $2017,4.1 \%{ }^{47}$ The differences might be due to geographical variation, study setting, methodological differences, limited sample size, differences in study period, and differences in TB control and prevention practice.

The high RR-MTB prevalence in different parts of Ethiopia, ${ }^{11,22,24}$ Congo, ${ }^{26}$ and India ${ }^{48}$ is explained by the included study participants who were suspected of MDRTB (relapse, defaulter, lost, and failure) rather than presumptive TB patients. The lowest RR-MTB reported in Ethiopia ${ }^{30}$ might be due to the study conducted was a community-based study, rather than a health service-based study. In comparison to our study, the higher RR-MTB reports from the Somali region, ${ }^{49}$ Pakistan, ${ }^{50}$ Bangladesh, ${ }^{51}$ and Togo ${ }^{27}$ might be explained by the variation in the study period and the scope to use Xpert for TB diagnosis (when the Xpert assay was started to be used, it was recommended only for patients with presumptive MDR-TB; but, later, the Xpert MTB/RIF assay was recommended for all TB suspected patients). 
In our study, RR-MTB had no significant association with gender. However, other studies conducted in different $\operatorname{areas}^{24,46,52}$ have reported more RR-MTB among females compared to males. In contrast to our findings, however, other studies conducted in different areas reported more RR-MTB infection among males than females. ${ }^{44,53,54}$ In the present study, age groups of $>75$ years were less infected by RR-MTB compared to the other age groups. But, other studies ${ }^{24,54}$ reported less prevalence of RRMTB between the age groups of 30-39 years. In our study, TB patients with previous treatment history (relapse, failure, default) were significantly associated with RR-MTB. This is in line with previous reports from different areas. ${ }^{11,46,52}$ As a result, coordinated efforts should be applied to treatment monitoring, limiting the transmission through the application of appropriate control and prevention methods to reduce the emergence of circulating drug-resistant MTB strains in the community.

In the current study, we tried to see the trend of MTB and RR-MTB for the last seven years. Accordingly, a higher prevalence of MTB was reported in 2017 and 2020 with a prevalence of $20.1 \%$ and $16.2 \%$, respectively. A high prevalence of RR-MTB was recorded in 2017 and 2019 accounting for $8.5 \%$ and $8 \%$, respectively. Even though the overall prevalence of both MTB and RR-MTB were low, there is no significant reduction from time to time. Thus, health programmers and stakeholders should give special attention and design a package in the national TB control program that addresses such areas where thousands of people are living in overcrowded areas.

\section{Limitations of the Study}

This study included a large sample size and is believed to give representative and updated information on the prevalence of MTB and RR-MTB to the regional and national governments. However, it has its own limitations. As the study was retrospective, first, we could not perform the genotypic confirmation of the Xpert positive MTB and RR-MTB. Second, only rifampicin resistance was analyzed and resistance to other anti-TB drugs was not detected because of the retrospective nature of the study. Third, there was a lack of important information and data like contact history of MDR-TB and TB, education, residence, types of non-sputum specimen used for TB detection, and living conditions of patients; thus, we were unable to show the associations between these factors with our outcome variables.

\section{Conclusion}

The overall prevalence of MTB and RR-MTB in this study was $11.8 \%$ and $5.1 \%$, respectively. Different age categories, being HIV positive, and previous TB treatment history (relapsed, failed, and defaulter) were significantly associated with high MTB. TB patients with previous treatment history (relapsed and failed) were also significantly associated with RR-MTB. As the prevalence of both MTB and RR-MTB was still high especially in patients with a history of previous treatment and no significant reduction from time to time, health programmers and stakeholders should give special attention and design a package in the national TB treatment, monitoring, and control program that addresses such areas where thousands of people live in overcrowded areas.

\section{Abbreviations}

AFB, Acid fast bacilli; AOR, Adjusted odds ratio; COR, Crude odds ratio; EPTB, Extra-pulmonary tuberculosis; HIV, Human immunodeficiency virus; MDR, Multi-drug resistance; MDRTB, Multidrug-resistant tuberculosis; MTB, Mycobacterium tuberculosis; PTB, Pulmonary tuberculosis; RIF, Rifampicin; RR-TB, Rifampicin-resistant tuberculosis; RR-MTB, Rifampicin-resistant Mycobacterium tuberculosis; SSA, SubSaharan Africa; TB, Tuberculosis; WHO, World Health Organization.

\section{Data Sharing Statement}

All data relevant to the study are included in the article and other raw data set used for analysis during the current study are available from the corresponding author on reasonable request.

\section{Acknowledgments}

The authors would like to acknowledge Dilla University Research and Dissemination Office for funding this research, Dilla University Referral Hospital medical director and all staff of department of medical laboratory for their cooperation in allowing the researchers to access the records and extract the data.

\section{Author Contributions}

All authors made a significant contribution to the work reported, whether that is in the conception, study design, execution, acquisition of data, analysis and interpretation, or in all these areas; took part in drafting, revising, or critically reviewing the article; gave final approval of the version to be published; have agreed on the journal to 
which the article has been submitted; and agree to be accountable for all aspects of the work.

\section{Funding}

This study was funded by Dilla University Research and Dissemination Office.

\section{Disclosure}

The authors declare that there are no conflicts of interest in this work.

\section{References}

1. Khan M, Raja Z, Ahmed H, Rauf A. A pattern of tuberculosis infection an overview. Pakistan J Biotechnol. 2019;16(3):153-161.

2. World Health Organization. Global status report on alcohol and health 2018. World Health Organization; 2019.

3. World Health Organization. Tracking universal health coverage: first global monitoring report. World Health Organization; 2015.

4. World Health Organization. Global tuberculosis report 2020: executive summary; 2020.

5. World Health Organization. Global tuberculosis report 2013. World Health Organization; 2013.

6. Migliori GB, Tiberi S, García-Basteiro AL, Duarte R. Tuberculosis and its future in the COVID-19 era: the pulmonology series 2021. Pulmonology. 2021;27:94-96.

7. Uplekar M, Weil D, Lonnroth K, et al. WHO's new end TB strategy. Lancet. 2015;385(9979):1799-1801. doi:10.1016/S0140-6736(15) 60570-0

8. Migliori GB, Dheda K, Centis R, et al. Review of multidrug-resistant and extensively drug-resistant TB: global perspectives with a focus on Sub-Saharan Africa. Trop Med Int Health. 2010;15(9):1052-1066. doi:10.1111/j.1365-3156.2010.02581.x

9. Zignol M, Gemert W, Falzon D, et al. Surveillance of antituberculosis drug resistance in the world: an updated analysis, 2007-2010. Bull World Health Organ. 2012;90:111-119. doi:10.2471/BLT.11.092585

10. World Health Organization. Rapid implementation of the Xpert MTB/RIF diagnostic test: technical and operational'How-to'; practical considerations. World Health Organization; 2011:9241501561.

11. Mulisa G, Workneh T, Hordofa N, Suaudi M, Abebe G, Jarso G. Multidrug-resistant Mycobacterium tuberculosis and associated risk factors in Oromia Region of Ethiopia. Int $J$ Infect Dis. 2015;39:57-61. doi:10.1016/j.ijid.2015.08.013

12. Girum T, Muktar E, Lentiro K, Wondiye H, Shewangizaw M. Epidemiology of multidrug-resistant tuberculosis (MDR-TB) in Ethiopia: a systematic review and meta-analysis of the prevalence, determinants and treatment outcome. Trop Dis Travel Med Vaccines. 2018;4(1):1-12. doi:10.1186/s40794-018-0065-5

13. Eshetie S, Gizachew M, Dagnew M, et al. Multidrug resistant tuberculosis in Ethiopian settings and its association with previous history of anti-tuberculosis treatment: a systematic review and meta-analysis. BMC Infect Dis. 2017;17(1):1-12. doi:10.1186/ s12879-017-2323-y

14. Bālaśelțān EYS, Macro O. Ethiopia demographic and health survey, 2005. Central Statistical Authority; 2006.

15. Wasihun AG, Hailu GG, Dejene TA. Prevalence of Mycobacterium tuberculosis (Rifampicin-Resistant MTB) and associated risk actors among pulmonary presumptive TB patients in Eastern Amhara, Ethiopia: 2015-2019. Infect Dis Ther. 2021;10:1299-1308.
16. Desta K, Asrat D, Lemma E, Gebeyehu M, Feleke B. Drug susceptibility of Mycobacterium tuberculosis isolates from smear negative pulmonary tuberculosis patients, Addis Ababa, Ethiopia. Ethiop $J$ Health Dev. 2008;22(2):212-215.

17. Nicol MP, Workman L, Isaacs W, et al. Accuracy of the Xpert MTB/ RIF test for the diagnosis of pulmonary tuberculosis in children admitted to hospital in Cape Town, South Africa: a descriptive study. Lancet Infect Dis. 2011;11(11):819-824. doi:10.1016/S14733099(11)70167-0

18. Kim CH, Hyun IG, Hwang YI, et al. Identification of Mycobacterium tuberculosis and rifampin resistance in clinical specimens using the Xpert MTB/RIF assay. Ann Clin Lab Sci. 2015;45(1):32-38.

19. Mulu W, Abera B, Yimer M, Hailu T, Ayele H, Abate D. Rifampicinresistance pattern of Mycobacterium tuberculosis and associated factors among presumptive tuberculosis patients referred to Debre Markos Referral Hospital, Ethiopia: a cross-sectional study. $B M C$ Res Notes. 2017;10(1):1-8.

20. Hordofa MW, Adela TB. Prevalence of Refampcin mono resistant Mycobacterium tuberculosis among suspected cases attending at Yirgalem hospital. Clin Med Res. 2015;4(3):75-78. doi:10.11648/j. cmr.20150403.13

21. Geleta DA, Megerssa YC, Gudeta AN, Akalu GT, Debele MT, Tulu KD. Xpert MTB/RIF assay for diagnosis of pulmonary tuberculosis in sputum specimens in remote health care facility. $B M C$ Microbiol. 2015;15(1):1-6. doi:10.1186/s12866-015-0566-6

22. Mehari K, Asmelash T, Hailekiros H, et al. Prevalence and factors associated with multidrug-resistant tuberculosis (MDR-TB) among presumptive MDR-TB patients in Tigray Region, Northern Ethiopia. Can J Infect Dis Med Microbiol. 2019;2019:1-8. doi:10.1155/2019/ 2923549

23. Adane K, Ameni G, Bekele S, Abebe M, Aseffa A. Prevalence and drug resistance profile of Mycobacterium tuberculosis isolated from pulmonary tuberculosis patients attending two public hospitals in East Gojjam zone, northwest Ethiopia. BMC Public Health. 2015;15(1):1-8. doi:10.1186/s12889-015-1933-9

24. Nigus D, Lingerew W, Beyene B, Tamiru A, Lemma M, Melaku M. Prevalence of multi drug resistant tuberculosis among presumptive multi drug resistant tuberculosis cases in Amhara National Regional State, Ethiopia. J Mycobac Dis. 2014;4(152):2161-1068. doi:10.4172/2161-1068.1000152

25. Tahiri L, Azzouzi H, Squalli G, Abourazzak F, Harzy T. Celiac disease causing severe osteomalacia: an association still present in Morocco! Pan Afr Med J. 2014;19. doi:10.11604/pamj.2014.19.43.2757

26. Farra A, Manirakiza A, Yambiyo BM, et al. editors. Surveillance of rifampicin resistance with GeneXpert MTB/RIF in the National Reference Laboratory for tuberculosis at the Institut Pasteur in Bangui, 2015-2017. In: Open Forum Infectious Diseases. US: Oxford University Press; 2019.

27. Dagnra A, Mlaga K, Adjoh K, Kadanga E, Disse K, Adekambi T. Prevalence of multidrug-resistant tuberculosis cases among HIV-positive and HIV-negative patients eligible for retreatment regimen in Togo using GeneXpert MTB/RIF. New Microbes New Infect. 2015;8:24-27. doi:10.1016/j.nmni.2015.09.001

28. Yin L, Qin G, Qian H-Z, et al. Continued spread of HIV among injecting drug users in southern Sichuan Province, China. Harm Reduct J. 2007;4(1):1-7. doi:10.1186/1477-7517-4-6

29. Sinshaw W, Kebede A, Bitew A, et al. Prevalence of tuberculosis, multidrug resistant tuberculosis and associated risk factors among smear negative presumptive pulmonary tuberculosis patients in Addis Ababa, Ethiopia. BMC Infect Dis. 2019;19(1):1-15. doi:10.1186/s12879-019-4241-7

30. Hamusse SD, Teshome D, Hussen MS, Demissie M, Lindtjørn B. Primary and secondary anti-tuberculosis drug resistance in Hitossa District of Arsi zone, Oromia regional state, Central Ethiopia. BMC Public Health. 2016;16(1):1-10. doi:10.1186/s12889-016-3210-y 
31. Biru D, Woldesemayat EM. Determinants of drug-resistant tuberculosis in southern Ethiopia: a case-control study. Infect Drug Resist. 2020;13:1823. doi:10.2147/IDR.S256536

32. Christopher PM, Widysanto A. GeneXpert Mycobacterium tuberculosis/Rifampicin assay for molecular epidemiology of Rifampicin-Resistant Mycobacterium tuberculosis in an urban setting of Banten Province, Indonesia. Int J Mycobacteriol. 2019;8(4):351. doi:10.4103/ijmy.ijmy_138_19

33. Odindo BO. Determinants of multi-drug resistant tuberculosis in patients in Homa Bay County Referral Hospital. Kenya: Maseno University; 2021.

34. Abebe G, Abdissa K, Abdissa A, et al. Relatively low primary drug resistant tuberculosis in southwestern Ethiopia. BMC Res Notes. 2012;5(1):1-6. doi:10.1186/1756-0500-5-225

35. Sa'ed HZ, Al-Jabi SW. Mapping the situation of research on coronavirus disease-19 (COVID-19): a preliminary bibliometric analysis during the early stage of the outbreak. BMC Infect Dis. 2020;20 (1): $1-8$.

36. Hordofa GM. Multi-drug resistant Mycobacterium tuberculosis and. BMC Res Notes. 2012;5(462):266.

37. Odum EP, Udi EO. Evaluation of cardiovascular risk factors in patients with chronic kidney disease. Port Harcourt Med J. 2017;11 (2):60. doi:10.4103/phmj.phmj_15_17

38. Snow K, Yadav R, Denholm J, Sawyer S, Graham S. Tuberculosis among children, adolescents and young adults in the Philippines: a surveillance report. Western Pac Surveill Response J. 2018;9 (4):16. doi:10.5365/wpsar.2017.8.4.011

39. Rahman A, Sahrin M, Afrin S, et al. Comparison of Xpert MTB/RIF assay and GenoType MTBDR plus DNA probes for detection of mutations associated with rifampicin resistance in Mycobacterium tuberculosis. PLoS One. 2016;11(4):e0152694. doi:10.1371/journal. pone. 0152694

40. Araya S, Negesso AE, Tamir Z. Rifampicin-resistant Mycobacterium tuberculosis among patients with presumptive tuberculosis in Addis Ababa, Ethiopia. Infect Drug Resist. 2020;13:3451. doi:10.2147/IDR. S263023

41. Lehours P, Siffré E, Mégraud F. DPO multiplex PCR as an alternative to culture and susceptibility testing to detect Helicobacter pylori and its resistance to clarithromycin. BMC Gastroenterol. 2011;11(1):1-5. doi:10.1186/1471-230X-11-112

42. Sekadde MP, Wobudeya E, Joloba ML, et al. Evaluation of the Xpert MTB/RIF test for the diagnosis of childhood pulmonary tuberculosis in Uganda: a cross-sectional diagnostic study. BMC Infect Dis. 2013;13(1):1-8. doi:10.1186/1471-2334-13-133

43. Chrisman CJ, Albuquerque P, Guimaraes AJ, Nieves E, Casadevall A. Phospholipids trigger Cryptococcus neoformans capsular enlargement during interactions with amoebae and macrophages. PLoS Pathog 2011;7(5):e1002047. doi:10.1371/journal.ppat.1002047
44. Tesfay K, Tesfay S, Nigus E, Gebreyesus A, Gebreegziabiher D, Adane K. More than half of presumptive multidrug-resistant cases referred to a tuberculosis referral laboratory in the Tigray region of Ethiopia are multidrug resistant. Int J Mycobacteriol. 2016;5 (3):324-327. doi:10.1016/j.ijmyco.2016.07.007

45. Ikuabe PO, Ebuenyi ID. Prevalence of rifampicin resistance by automated Genexpert rifampicin assay in patients with pulmonary tuberculosis in Yenagoa, Nigeria. Pan Afr Med J. 2018;29. doi:10.11604/ pamj.2018.29.204.14579

46. Tilahun M, Ameni G, Desta K, et al. Molecular epidemiology and drug sensitivity pattern of Mycobacterium tuberculosis strains isolated from pulmonary tuberculosis patients in and around Ambo Town, Central Ethiopia. PLoS One. 2018;13(2):e0193083. doi:10.1371/journal.pone.0193083

47. Barreira D. The challenges to eliminating tuberculosis in Brazil. Epidemiologia e Serviços de Saúde. 2018;27:e00100009.

48. Kaur R, Jindal N, Arora S, Kataria S. Epidemiology of rifampicin resistant tuberculosis and common mutations in rpoB gene of Mycobacterium tuberculosis: a retrospective study from six districts of Punjab (India) using Xpert MTB/RIF assay. J Lab Physicians. 2016;8(2):96. doi:10.4103/0974-2727.180789

49. Mehmood A, Abdallah K, Khandekar S, et al. Expression of extra-cellular levansucrase in Pseudomonas syringae is controlled by the in planta fitness-promoting metabolic repressor HexR. BMC Microbiol. 2015;15(1):1-11. doi:10.1186/s12866-015-0349-0

50. Reddy R, Alvarez-Uria G. Molecular epidemiology of Rifampicin resistance in Mycobacterium tuberculosis using the GeneXpert MTB/ RIF assay from a rural setting in India. J Pathog. 2017;2017:1-5. doi: $10.1155 / 2017 / 6738095$

51. Zar HJ, Connell TG, Nicol M. Diagnosis of pulmonary tuberculosis in children: new advances. Expert Rev Anti Infect Ther. 2010;8 (3):277-288. doi:10.1586/eri.10.9

52. Arega B, Menbere F, Getachew Y. Prevalence of rifampicin resistant Mycobacterium tuberculosis among presumptive tuberculosis patients in selected governmental hospitals in Addis Ababa, Ethiopia. BMC Infect Dis. 2019;19(1):307. doi:10.1186/s12879-0193943-1

53. Hailu AG. Prevalence of Mycobacterium tuberculosis (RifampicinResistant MTB) and associated risk actors among pulmonary presumptive TB patients in Eastern Amhara, Ethiopia: 2015-2019; 2021.

54. Toungoussova OS, Caugant DA, Sandven P, Mariandyshev AO, Bjune G. Drug resistance of Mycobacterium tuberculosis strains isolated from patients with pulmonary tuberculosis in Archangels, Russia. Int J Tuberc Lung Dis. 2002;6(5):406-414.
Infection and Drug Resistance

\section{Publish your work in this journal}

Infection and Drug Resistance is an international, peer-reviewed openaccess journal that focuses on the optimal treatment of infection (bacterial, fungal and viral) and the development and institution of preventive strategies to minimize the development and spread of resistance. The journal is specifically concerned with the epidemiology of antibiotic resistance and the mechanisms of resistance development and diffusion in both hospitals and the community. The manuscript management system is completely online and includes a very quick and fair peerreview system, which is all easy to use. Visit http://www.dovepress.com/ testimonials.php to read real quotes from published authors. 